\title{
Effects of vitamin D with or without calcium on pathological ossification: A retrospective clinical study
}

\author{
LIBIN LIANG ${ }^{1}$, TONG TONG ${ }^{2}$, LUYANG QIN ${ }^{3}$, JING XIE $^{4}$, YANPING XU ${ }^{1}$, YUGUO QIN ${ }^{1}$, \\ LANSEN ZHANG $^{1}$, DEHUI LIU ${ }^{1}$, XIAOYUN NIU ${ }^{1}$ and XINGLONG TONG ${ }^{1}$ \\ ${ }^{1}$ Hebei Xinglong Institute of Pharmaceutical and Medical Science, Shijiazhuang, Hebei 050091; \\ ${ }^{2}$ Department of Spine Surgery, The Third Hospital of Hebei Medical University, Shijiazhuang, Hebei 050051; \\ ${ }^{3}$ School of Clinical Medicine, North China University of Science and Technology, Tangshan, Hebei 063210; \\ ${ }^{4}$ Graduate School, Hebei Medical University, Shijiazhuang, Hebei 050017, P.R. China
}

Received December 7, 2021; Accepted January 26, 2022

DOI: $10.3892 / \mathrm{etm} .2022 .11214$

\begin{abstract}
Vitamin D protects against the development and severity of several rheumatic diseases. However, the effect of vitamin D on the pathological ossification associated with rheumatic diseases remains unknown. The present retrospective study analyzed the clinical outcomes of vitamin D without calcium compared with vitamin $D$ with calcium on pathological ossification in joints and ligaments. Data were collected from patients who were diagnosed with osteoarthritis, rheumatoid arthritis or spondylarthritis, and the presence of pathological ossification in joints or ligaments was confirmed by X-ray, computed tomography or magnetic resonance imaging examination. A total of 2,965 patients aged 18-75 years old were included, among who, 1,725 were included in the vitamin D alone group and 1,240 in the vitamin $\mathrm{D}$ with calcium group. Vitamin D was administered intramuscularly (300,000 IU) once every 7-10 days, 4-6 times in total. Patients who ingested an oral calcium supplement $(1,000 \mathrm{mg} / \mathrm{day} ; \geq 5$ days/week $)$ were considered the vitamin D with calcium group. The clinical outcome was evaluated based on the imaging changes of pathological ossification, which were classified as alleviation, aggravation and unchanged. The bone mineral density (BMD) was determined, and the calcium concentration in the serum and urine was measured. The results revealed that vitamin D alone alleviated pathological ossification, while vitamin D combined with calcium aggravated pathological ossification in the majority of patients $(\mathrm{P}<0.0001)$ independent of disease type and patient age. BMD measurements demonstrated a decreasing trend in the vitamin $\mathrm{D}$ alone group, whereas they exhibited an increasing trend in the vitamin D combined with
\end{abstract}

Correspondence to: Dr Xinglong Tong, Hebei Xinglong Institute of Pharmaceutical and Medical Science, 645 South Hongqi Street, Shijiazhuang, Hebei 050091, P.R. China

E-mail: xinglongtong@hotmail.com

Key words: vitamin D, calcium supplementation, pathological ossification, bone mineral density calcium group. The urine calcium concentration increased after vitamin D treatment alone. Therefore, it was concluded that vitamin D exerted both pro-resorptive and anti-resorptive actions on pathological ossification. The bidirectional action of vitamin D on bone metabolism may depend on exogenous calcium supplementation.

\section{Introduction}

Osteoarthritis (OA) is a common degenerative disease of the joints; it is characterized by the destruction of articular cartilage, often accompanied by hypertrophy of chondrocytes and calcification, resulting in the formation of new bones (osteophytes) or cartilage ossification bands on the edge of the joints (1). This pathological ossification is also common in other rheumatic diseases, including rheumatoid arthritis (RA) and spondylarthritis (SA) (2). In addition, ectopic osteogenesis is frequently observed in spinal ligaments, including the posterior longitudinal ligament and ligamentum flavum (3). The abnormal ossification of joints clinically causes pain, movement disorders and joint deformation, and the ossification of spine ligaments compresses the spinal cord or nerve roots, which leads to various degrees of neurological symptoms (3). Besides surgical treatment, no effective strategy has been indicated to promote the regression of abnormal ossification.

Vitamin D plays a pivotal role in maintaining calcium and phosphate homeostasis, as well as in regulating bone metabolism; it undergoes bioconversion to an active form called 1,25-dyhydroxyvitamin $\left.\mathrm{D}\left[1,25(\mathrm{OH})_{2} \mathrm{D}\right)\right]$, which elicits its biological functions through vitamin D receptor (VDR) (4). Activation of VDR promotes absorption or resorption of calcium and phosphorus in the intestine and renal tubules, and regulates parathyroid hormone secretion (4). These functions can indirectly regulate bone growth and mineralization. In addition, vitamin $\mathrm{D}$ directly acts on bone growth and mineralization, as well as in bone remodeling (5). Accumulating evidence has demonstrated that vitamin D exerts opposing effects on bones, such as anti-resorptive and pro-resorptive effects (6). Previous in vitro and in vivo experiments have demonstrated that higher concentrations/doses of vitamin D stimulate osteoclastic bone resorption (6). In addition to 
regulating calcium and phosphate metabolism, vitamin D is also known as an immunomodulatory hormone, and thus aids in the protection against the development of various severe rheumatic diseases, including OA, RA and SA (7-9). However, the effect of vitamin $D$ on pathological ossification associated with rheumatic diseases remains unknown. Based on the pro-resorptive action of vitamin $\mathrm{D}$, it was speculated that high doses of vitamin $\mathrm{D}$ may produce ameliorative effects on pathological ossification. In addition, calcium is usually employed in combination with vitamin D for the treatment of rheumatic diseases. Calcium directly promotes osteoblast to osteocyte transition and thus stimulates bone mineralization (10). Whether calcium supplementation affects the action mode of vitamin $\mathrm{D}$ is also unknown. The aim of the present study was to compare the clinical outcomes of using vitamin D alone and vitamin D with simultaneous calcium supplementation on the pathological ossification associated with several rheumatic diseases.

\section{Materials and methods}

Study design. In the present retrospective study, data were collected from patients of either sex who had been diagnosed with OA, RA or SA at an age range of 18 to 75 years, in whom abnormal ossification in the joints and vertebral bodies, as well as in the spinal ligaments (posterior longitudinal ligament or ligamentum flavum), was confirmed by X-ray, computed tomography or magnetic resonance imaging examination. Patients were examined in Qiaoxi Tong-Xinglong Western Medicine Clinic (Shijiazhuang, China) between January 2010 and December 2019. All patients in the present study received 300,000 IU vitamin D intramuscularly, once every 7-10 days, 4-6 times in total. Patients receiving additional oral calcium administration (1,000 mg/day; $\geq 5$ days/week) were defined as the vitamin $\mathrm{D}$ with calcium group. The remaining patients were defined as the vitamin D only group. The total period of treatment was 1-2 months. All patients provided written informed consent before receiving vitamin D. Exclusion criteria prior to and during the study included the following: i) Medical conditions or disorders that influence bone mineral metabolism; ii) obvious clinical symptoms associated with severe organ diseases (including heart, liver, kidney, lung, digestive tract and thyroid) or other metabolic diseases, which required specific therapeutic interventions for $>1$ week; and iii) patients who did not receive $\geq 4$ injections of vitamin $D$. The imaging examination was performed before treatment and within 1 month of treatment. The primary endpoint was evaluated based on the imaging changes of pathological ossification, which were classified into three types of outcomes: i) Alleviation; ii) aggravation; and iii) unchanged. Evaluation of imaging changes was performed by two radiologists and an orthopedic surgeon.

Bone mineral density (BMD) determination. BMD was determined in 141 patients in the vitamin D alone group and 135 patients in the vitamin $\mathrm{D}$ and calcium group at the lumbar spine (L1-L4) and the left femoral neck before and after vitamin $\mathrm{D}$ treatment via dual energy X-ray absorptiometry (Hologic Discovery-A; Hologic Canada ULC); as a measurement of precision, the coefficient of variation was $<1 \%$.
Biochemical analysis. The levels of calcium and phosphorus in serum and urine were determined in 31 patients in the vitamin $\mathrm{D}$ alone group and 32 patients in the vitamin $\mathrm{D}$ and calcium group. Blood and urine samples were collected in the morning after overnight fasting, and serum and urinary concentrations of total calcium and phosphorus were measured using an automatic biochemical analyzer (AU5400; Olympus Corporation).

Safety assessment. Adverse reactions reported by the patients were collected. Changes in physiological parameters, including vital signs and electrocardiogram readings, were recorded in 40 patients in the vitamin D alone group and 36 patients in the vitamin $\mathrm{D}$ and calcium group; biochemical blood analyses including alanine aminotransferase (ALT), aspartate aminotransferase (AST), creatine kinase (CK), total protein (TP), urea nitrogen (BUN) were performed in 18 patients in the vitamin $\mathrm{D}$ alone group and 17 patients in the vitamin $\mathrm{D}$ and calcium group. In addition, some patients underwent ultrasound examination of abdominal organs and neck vasculature before and after treatment.

Statistical analysis. The number of patients (percentage) were used to describe patients' clinical characteristics and the $\chi^{2}$ test was applied to evaluate the efficacy difference between two groups. Quantitative data including BMD, biochemical parameters and levels of calcium and phosphorus were presented as the mean \pm SD unless stated otherwise and the differences were evaluated using paired Student's t-test for single repeated measurements or unpaired Student's t-test for comparison of changes between two groups. Data analysis was performed with SPSS version 20 (IBM Corp.). $\mathrm{P}<0.05$ was considered to indicate a statistically significant difference.

\section{Results}

Clinical outcomes. A total of 2,965 patients were included in the present study, among who, 1,725 were in the vitamin D alone group and 1,240 were in the vitamin D with calcium group. Joint pathological ossification primarily occurred in the cervical and lumbar spine, knee, elbow, ankle, wrist and finger joints. Spinal ligament (posterior longitudinal ligament and ligamentum flavum) ossification was also observed. The outcomes of the two regimens on the pathological ossification associated with OA and other types of diseases were separately analyzed. There was no significant difference in sex, age or ossification sites between the vitamin D alone and vitamin D with calcium groups (Table I). Considering the possible impact of age-related dietary calcium intake on the study, the populations $<40$ and $\geq 40$ years of age were separately analyzed. In the population $<40$ years of age, 54\% (123/228) of patients with OA in the vitamin D alone group exhibited alleviation, 18\% (42/228) exhibited aggravation and the remaining $28 \%$ (63/228) presented with unchanged symptoms (Table II). By contrast, only 3\% (5/185) of subjects in the vitamin D with calcium group showed alleviation [risk ratio (RR), 1.741; 95\% confidence interval (CI), 1.585-1.912; $\mathrm{P}<0.0001]$, while $66 \%(122 / 185)$ showed aggravation (RR, 0.464; 95\% CI, 0.352-0.611; $\mathrm{P}<0.0001)$ and 31\% (58/185) presented with unchanged symptoms (RR, 0.943; 95\% CI, 0.779-1.143; 
Table I. Baseline characteristics of patients included in the vitamin D alone $(n=1,725)$ and vitamin $D$ with calcium $(n=1,240)$ groups.

\begin{tabular}{|c|c|c|}
\hline Characteristic & Vitamin D alone, n (\%) & Vitamin D + calcium, n (\%) \\
\hline \multicolumn{3}{|l|}{ Sex } \\
\hline Male & $850(49.3)$ & $616(49.7)$ \\
\hline Female & $875(50.7)$ & $624(50.3)$ \\
\hline \multicolumn{3}{|l|}{ Age } \\
\hline $18-39$ & $555(32.2)$ & $459(37.0)$ \\
\hline $40-75$ & $1,170(67.8)$ & $781(63.0)$ \\
\hline Osteoarthritis & $837(48.5)$ & $451(36.4)$ \\
\hline Cervical vertebra & $237(13.7)$ & $88(7.1)$ \\
\hline Lumbar vertebra & $112(6.5)$ & $94(7.6)$ \\
\hline Knee joint & $140(8.1)$ & $78(6.3)$ \\
\hline Elbow joint & $61(3.5)$ & $32(2.6)$ \\
\hline Ankle joint & $29(1.7)$ & $21(1.7)$ \\
\hline Wrist joint & $28(1.6)$ & $15(1.2)$ \\
\hline Knuckle & $17(1.0)$ & $12(1.0)$ \\
\hline Posterior longitudinal ligament & $118(6.8)$ & $55(4.4)$ \\
\hline Ligamentum flavum & $95(5.5)$ & $56(4.5)$ \\
\hline Other rheumatic diseases & $888(51.5)$ & $789(63.6)$ \\
\hline Cervical vertebra & $135(7.8)$ & $97(7.8)$ \\
\hline Lumbar vertebra & $164(9.5)$ & $132(10.6)$ \\
\hline Knee joint & $140(8.1)$ & $146(11.8)$ \\
\hline Elbow joint & $99(5.7)$ & $121(9.8)$ \\
\hline Ankle joint & $136(7.8)$ & $105(8.5)$ \\
\hline Wrist joint & $98(5.7)$ & $89(7.2)$ \\
\hline Knuckle & $116(6.7)$ & $99(8.0)$ \\
\hline
\end{tabular}

$\mathrm{P}=0.6039)$. Similar outcomes were obtained in patients with RA and SA. Vitamin D alone resulted in 64\% (209/327) alleviation, while vitamin $\mathrm{D}$ combined with calcium aggravated the ossification in 69\% (188/274) (RR, 0.309; 95\% CI, 0.229-0.416; $\mathrm{P}<0.0001)$. Comparable therapeutic outcomes on pathological ossification in the population $\geq 40$ years of age were observed. For OA-associated pathological ossification, treatment with vitamin $\mathrm{D}$ alone resulted in 70\% (426/609) alleviation, 10\% (59/609) aggravation, and $20 \%(124 / 609)$ unchanged; whereas vitamin D combined with calcium led to $64 \%$ (171/266) aggravation (RR, 0.369; 95\% CI, 0.295-0.461; P<0.0001), 7\% (18/266) alleviation (RR, 1.379; 95\% CI, 1.314-1.446; P<0.0001), and 30\% (77/266) unchanged (RR, 0.886; 95\% CI, 0.788-0.997; P=0.0357) (Table III). Similar results were observed for RA- and SA-associated pathological ossification (Table III). The representative imaging alterations for 'alleviation' in the vitamin D alone group and 'aggravation' in the vitamin D with calcium group are demonstrated in Figs. S1 and S2, respectively. The results indicated that vitamin $\mathrm{D}$ alone promoted the resorption of abnormal ossification, whereas vitamin D combined with calcium aggravated the ossification in the majority of patients, independently of the disease type.

It was noted that, for OA-associated abnormal ossification, vitamin D alone treatment resulted in $70 \%$ alleviation in the population of $\geq 40$ years of age (Table III), which was significantly higher than that observed in patients $<40$ years of age $(54 \%$; Table II) $(\mathrm{P}<0.05)$. This may be due to a higher calcium intake in the diet of young individuals compared with that in the elderly (11). In addition, the treatment regimen of vitamin D was slightly different among patients who received intramuscular injection once every 7 to 10 days for 4 to 6 times since it was not applicable to choose patients with exact same and times in this retrospective analysis. This study revealed that the slight variance of vitamin D application time and frequency did not affect the outcomes of treatments (data not shown).

BMD measurement results. Next, the effects of vitamin D with or without calcium on BMD were evaluated. Considering the possible differences in BMD in females, particularly in postmenopausal females, the data were separately analyzed according to patient sex and age. As presented in Table IV, there was no significant difference in BMD at the lumbar spine or femoral neck before and after treatment with vitamin D with or without calcium among different sex or age groups. However, vitamin D alone produced a decreasing trend in BMD in different sex and age groups, whereas vitamin D with calcium demonstrated an increasing trend in BMD.

Biochemical analysis results. Biochemical analysis indicated that the serum calcium concentration did not change after the 
Table II. Clinical outcomes of vitamin D alone $(\mathrm{n}=555)$ and vitamin $\mathrm{D}$ with calcium $(\mathrm{n}=459)$ groups on the pathological ossification in patients $<40$ years old.

\begin{tabular}{llccrrr}
\hline Disease & Outcome & $\begin{array}{c}\text { Vitamin D alone, } \\
\text { n/total n (\%) }\end{array}$ & $\begin{array}{c}\text { Vitamin D + calcium, } \\
\text { n/total n (\%) }\end{array}$ & RR & $95 \%$ CI & P-value \\
\hline Osteoarthritis & Alleviation & $123 / 228(54)$ & $5 / 185(3)$ & 1.741 & $1.585-1.912$ & $<0.0001$ \\
& Aggravation & $42 / 228(18)$ & $122 / 185(66)$ & 0.464 & $0.352-0.611$ & $<0.0001$ \\
& Unchanged & $63 / 228(28)$ & $58 / 185(31)$ & 0.943 & $0.779-1.143$ & 0.6039 \\
Other rheumatic & Alleviation & $209 / 327(64)$ & $12 / 274(4)$ & 1.738 & $1.605-1.882$ & $<0.0001$ \\
diseases & Aggravation & $38 / 327(12)$ & $188 / 274(69)$ & 0.309 & $0.229-0.416$ & $<0.0001$ \\
& Unchanged & $80 / 327(24)$ & $74 / 274(27)$ & 0.955 & $0.807-1.130$ & 0.5881 \\
\hline
\end{tabular}

$\mathrm{RR}$, risk ratio; CI, confidence interval.

Table III. Clinical outcomes of vitamin D alone $(n=1,170)$ and vitamin D with calcium $(n=781)$ groups on the pathological ossification in patients $\geq 40$ years old.

\begin{tabular}{llcccrr}
\hline Disease & Outcome & $\begin{array}{c}\text { Vitamin D alone, } \\
\text { n/total n (\%) }\end{array}$ & $\begin{array}{c}\text { Vitamin D + calcium, } \\
\text { n/total n (\%) }\end{array}$ & RR & $95 \%$ CI & P-value \\
\hline Osteoarthritis & Alleviation & $426 / 609(70)$ & $18 / 266(7)$ & 1.379 & $1.314-1.446$ & $<0.0001$ \\
& Aggravation & $59 / 609(10)$ & $171 / 266(64)$ & 0.369 & $0.295-0.461$ & $<0.0001$ \\
& Unchanged & $124 / 609(20)$ & $77 / 266(30)$ & 0.886 & $0.788-0.997$ & 0.0357 \\
Other rheumatic diseases & Alleviation & $362 / 561(65)$ & $33 / 515(6)$ & 1.758 & $1.648-1.875$ & $<0.0001$ \\
& Aggravation & $52 / 561(9)$ & $337 / 515(65)$ & 0.256 & $0.198-0.332$ & $<0.0001$ \\
& Unchanged & $147 / 561(26)$ & $145 / 515(28)$ & 0.966 & $0.850-1.097$ & 0.5978 \\
\hline
\end{tabular}

$\mathrm{RR}$, risk ratio; CI, confidence interval.

administration of vitamin D alone in males or females, but the urine calcium concentration was significantly increased in both sexes (both $\mathrm{P}<0.05$ ), suggesting an increase in calcium excretion (Table V). Vitamin D alone did not significantly alter serum or urinary phosphorus levels. Vitamin D with calcium exhibited no significant effect on calcium or phosphorus levels in either the serum or urine (Table V).

Drug safety assessment. Due to the high dose of vitamin D used in the present study, its safety was evaluated. No serious adverse reactions were reported from any of the participants during treatment or the follow-up period of observation. Changes in physiological parameters, including body temperature, heart rate, RR, blood pressure and blood glucose, and adverse reactions such as arrhythmias and cardiac ischemia, are presented in Table SI. The number of subjects with positive changes or with adverse reactions did not notably differ between the two groups. In addition, subjects in the vitamin D with or without calcium groups exhibited no significant alterations in the blood biochemical parameters including ALT, AST, CK,TP and BUN, which reflecting functional changes in the heart, liver and kidney (Table SII). A total of 18 patients (10 males and 8 females) in the vitamin D alone and 17 patients ( 8 males and 9 females) in the vitamin $\mathrm{D}$ with calcium group underwent ultrasound examination of the abdominal organs and neck vasculature before and after treatment in order to detect heterotopic ossification of the soft tissues. There was no new ossification in the abdominal organs or neck vessels of the patients treated with vitamin D alone, whereas abdominal ultrasound examination of subjects treated with vitamin D with calcium revealed an aggravated prostate ossification in one subject, a novel prostate ossification in another subject and a suspected kidney stone in a female patient. Cervical vascular ultrasound examination revealed a calcified plaque of the common carotid artery in a male patient and an aggravated calcified plaque of an internal carotid artery in a female patient.

\section{Discussion}

The main findings of the present clinical study were that vitamin D alone produced an improvement in the pathological ossification of joints or ligaments, whereas vitamin D in combination with calcium exhibited an opposing clinical outcome with aggravation of this ossification. Similar results were observed in subjects with different ages and were irrespective of the disease type. In addition, vitamin $D$ alone tended to reduce BMD in normal bone, while vitamin $\mathrm{D}$ with calcium tended to increase BMD. These results indicated that a high dose of vitamin $\mathrm{D}$ alone may have pro-resorptive action, whereas vitamin D combined with a calcium supplement may promote osteogenesis. 
Table IV. Bone mineral density measurement results.

\begin{tabular}{|c|c|c|c|c|}
\hline \multirow[b]{2}{*}{ Group } & \multicolumn{2}{|c|}{ Vitamin D alone } & \multicolumn{2}{|c|}{ Vitamin D + calcium } \\
\hline & Before & After & Before & After \\
\hline \multicolumn{5}{|l|}{$<40$ years } \\
\hline \multicolumn{5}{|l|}{ Male $^{\mathrm{a}}$} \\
\hline Lumbar spine & $0.997 \pm 0.084$ & $0.993 \pm 0.083^{\mathrm{e}}$ & $0.967 \pm 0.080$ & $0.971 \pm 0.080^{\mathrm{e}}$ \\
\hline Left femoral neck & $0.624 \pm 0.088$ & $0.620 \pm 0.086^{\mathrm{e}}$ & $0.597 \pm 0.069$ & $0.609 \pm 0.052^{\mathrm{e}}$ \\
\hline \multicolumn{5}{|l|}{ Female $^{\mathrm{b}}$} \\
\hline Lumbar spine & $0.932 \pm 0.086$ & $0.929 \pm 0.096^{\mathrm{e}}$ & $0.928 \pm 0.090$ & $0.933 \pm 0.087^{\mathrm{e}}$ \\
\hline Left femoral neck & $0.608 \pm 0.074$ & $0.602 \pm 0.072^{\mathrm{e}}$ & $0.594 \pm 0.052$ & $0.610 \pm 0.043^{\mathrm{e}}$ \\
\hline \multicolumn{5}{|l|}{$\geq 40$ years } \\
\hline \multicolumn{5}{|l|}{ Male $^{c}$} \\
\hline Lumbar spine & $0.985 \pm 0.070$ & $0.977 \pm 0.058^{\mathrm{e}}$ & $0.929 \pm 0.081$ & $0.938 \pm 0.081^{\mathrm{e}}$ \\
\hline Left femoral neck & $0.586 \pm 0.093$ & $0.574 \pm 0.083^{\mathrm{e}}$ & $0.565 \pm 0.079$ & $0.573 \pm 0.073^{\mathrm{e}}$ \\
\hline \multicolumn{5}{|l|}{ Female $^{\mathrm{d}}$} \\
\hline Lumbar spine & $0.931 \pm 0.081$ & $0.915 \pm 0.100^{\mathrm{e}}$ & $0.918 \pm 0.094$ & $0.928 \pm 0.090^{\mathrm{e}}$ \\
\hline Left femoral neck & $0.583 \pm 0.064$ & $0.575 \pm 0.064^{\mathrm{e}}$ & $0.557 \pm 0.078$ & $0.562 \pm 0.076^{\mathrm{e}}$ \\
\hline
\end{tabular}

Table V. Concentrations of calcium and phosphorus in serum and urine.

\begin{tabular}{|c|c|c|c|c|}
\hline \multirow[b]{2}{*}{ Group } & \multicolumn{2}{|c|}{ Calcium, mM } & \multicolumn{2}{|c|}{ Phosphorus, mM } \\
\hline & Before & After & Before & After \\
\hline \multicolumn{5}{|c|}{ Vitamin D alone } \\
\hline \multicolumn{5}{|c|}{ Male $(n=17)$} \\
\hline Serum & $2.32 \pm 0.15$ & $2.34 \pm 0.11$ & $1.13 \pm 0.24$ & $1.12 \pm 0.20$ \\
\hline Urine & $1.58 \pm 0.72$ & $2.65 \pm 1.18^{\mathrm{a}}$ & $7.46 \pm 1.30$ & $7.16 \pm 1.28$ \\
\hline \multicolumn{5}{|c|}{ Female $(n=14)$} \\
\hline Serum & $2.36 \pm 0.14$ & $2.36 \pm 0.11$ & $1.13 \pm 0.24$ & $1.20 \pm 0.22$ \\
\hline Urine & $1.63 \pm 0.71$ & $2.98 \pm 1.13^{\mathrm{a}}$ & $7.03 \pm 1.45$ & $7.00 \pm 1.18$ \\
\hline \multicolumn{5}{|c|}{ Vitamin D + calcium } \\
\hline \multicolumn{5}{|c|}{ Male $(n=16)$} \\
\hline Serum & $2.36 \pm 0.13$ & $2.40 \pm 0.11$ & $1.19 \pm 0.25$ & $1.14 \pm 0.24$ \\
\hline Urine & $1.52 \pm 0.82$ & $1.55 \pm 0.68$ & $6.99 \pm 1.55$ & $6.80 \pm 1.20$ \\
\hline \multicolumn{5}{|c|}{ Female $(n=16)$} \\
\hline Serum & $2.33 \pm 0.13$ & $2.39 \pm 0.10$ & $1.19 \pm 0.14$ & $1.15 \pm 0.11$ \\
\hline Urine & $1.60 \pm 0.82$ & $1.71 \pm 0.94$ & $6.45 \pm 1.63$ & $6.41 \pm 1.52$ \\
\hline
\end{tabular}

${ }^{\mathrm{a}} \mathrm{P}<0.05$ vs. before.

At present, the direct action of the VDR in bone tissue is not well understood. It is widely accepted that vitamin D is important for bone growth, as its deficiency can lead to osteomalacia and rickets $(4,5)$. However, the direct effects of vitamin $\mathrm{D}$ on bone remain under debate. A previous study has demonstrated that $1,25(\mathrm{OH})_{2} \mathrm{D}$ stimulates osteoclast formation in the co-culture of mouse osteoblastic and hematopoietic cells (12). Osteoclast precursors express receptor activator of nuclear factor- $\kappa \mathrm{B}(\mathrm{RANK})$ and they recognize RANK ligand (RANKL), and differentiate into osteoclasts (13). In addition, $1,25(\mathrm{OH})_{2} \mathrm{D}$ increases RANKL expression in osteoblast-lineage cells, thus stimulating bone resorption through VDR expressed in osteoblast-lineage cells (14-16). Previous experiments have revealed that high-dose administration of 
$1,25(\mathrm{OH})_{2} \mathrm{D}$ inhibits the mineralization of osteoblasts $(17,18)$. These findings have suggested that a high dose of vitamin D may suppress osteoblastogenesis and stimulate bone resorption. It is generally accepted that the molecular mechanisms underlying pathological or ectopic ossification of joint or soft tissues are similar to those regulating the physiological ossification of skeletal tissues (1). Consistent with the aforementioned results, the present study revealed that a high dose of vitamin D alone alleviated pathological ossification of the joints and ligaments, with a trend in the reduction of BMD in normal bone. These results suggest that pathological ossification may be more sensitive to the pro-resorptive action of vitamin D than normal bone. It is known that vitamin D promotes bone resorption and can increase blood calcium levels (15). However, the present study did not find a significant increase in blood calcium; only a higher urine calcium concentration was observed. The increased urinary calcium excretion may maintain blood calcium homeostasis.

Notably, the present study revealed that vitamin D in combination with calcium supplementation resulted in the deterioration of pathological ossification, with a tendency for increased BMD in normal bone, while calcium supplementation reversed the action model of vitamin $\mathrm{D}$, namely from a pro-resorptive to an anti-resorptive effect. The mechanism underlying the regulation of exogenous calcium supplementation on the bidirectional effect of vitamin D is unknown. Previous evidence has demonstrated that calcium per se promotes osteoblast to osteocyte differentiation, inhibits the activation of osteoclasts and promotes bone mineralization in a concentration-dependent manner $(10,19)$. It can be hypothesized that in the presence of a sufficient quantity of calcium supplement, extraosseous action of vitamin D such as the promotion of the intestinal absorption of calcium and renal calcium resorption may make it predisposed to promote osteogenesis and bone mineralization. In addition, the results may also be secondary responses to the change of cytokines, since vitamin D has an immunomodulatory action (7-9). One of the limitations to the present study is that serum levels of inflammatory cytokines were not determined. The detailed mechanism underlying the bidirectional effect of vitamin D requires further elucidation.

Regarding the safety of vitamin D administration, the present study showed that 300,000 IU vitamin D administered intramuscularly every 7-10 days for a total of 4-6 times did not induce serious adverse reactions in any of the subjects. Vitamin D toxicity has been previously observed in patients who received multiple intramuscular injections of vitamin D, each containing 600,000 IU vitamin D (20). Thus, safety should be considered for the prolonged usage of vitamin D.

In conclusion, to the best of our knowledge, the present study demonstrated for the first time that vitamin D alone or in combination with calcium exhibited ameliorative or deteriorative effects, respectively, on pathological ossification. Although the mechanism by which vitamin D exerted biphasic and opposing effects on bone remains to be clarified, the results of the present study provided an important reference for the treatment of pathological ossification-related diseases and osteoporosis. Pathological ossification is an important feature in OA progression $(1,21)$. Inhibiting pathological ossification represents a potential therapeutic target in the management of OA. The present results suggest that vitamin D alone could promote the resorption of abnormal ossification and thus alleviate clinical symptoms, such as pain caused by bone spurs and the prevention of exercise. However, the balance of benefits and risks should be considered. The present study demonstrated that the treatment period should not exceed 2 months so that it has less impact on normal bone BMD. In addition, although vitamin D is widely used in the treatment of osteoporosis and the prevention of fractures from falls, clinical trials of various cohort sizes have so far failed to obtain consistent positive results (22-24). Previous studies in which vitamin D was administered as bolus doses reported significant increases in fractures and falls (25). A recent study indicated that 3 years of high-dose vitamin D supplementation (400, 4,000 and 10,000 IU) in healthy, vitamin D-sufficient individuals aged 55-70 years old resulted in a negative dose-response association with bone density and strength (26). The findings of the present study provide an explanation for the inconsistent or even contrary clinical outcomes of vitamin D treatment. It is necessary to optimize the dose, protocol and use of simultaneous calcium supplementation when applying vitamin D for the treatment of osteoporosis.

\section{Acknowledgements}

Not applicable.

\section{Funding}

No funding was received.

\section{Availability of data and materials}

The datasets used and/or analyzed during the current study are available from the corresponding author on reasonable request.

\section{Authors' contributions}

LL participated in the data collection and analysis. TT participated in the assessment of images. LQ and JX participated in the data analysis, and YX, YQ, LZ, DL and XN participated in acquisition of patients' data. XT designed and conceptualized the study, drafted the manuscript and contributed to interpretation of the data. LL and XT confirm the authenticity of all the raw data. All authors have read and approved the final version of the manuscript.

\section{Ethics approval and consent to participate}

The present study was approved by the Ethics Committee of Hebei Xinglong Institute of Pharmaceutical and Medical Science (Shijiazhuang, China; approval no. XLEC200901). Written informed consent was obtained from all patients prior to treatment.

\section{Patient consent for publication}

Written informed consent for the publication of any data and accompanying images was obtained from the patients. 


\section{Competing interests}

The authors declare that they have no competing interests.

\section{References}

1. Yan JF, Qin WP, Xiao BC, Wan QQ, Tay FR, Niu LN and Jiao K: Pathological calcification in osteoarthritis: An outcome or a disease initiator? Biol Rev Camb Philos Soc 95: 960-985, 2020.

2. Taylor AM: Metabolic and endocrine diseases, cartilage calcification and arthritis. Curr Opin Rheumatol 25: 198-203, 2013.

3. Furukawa K: Pharmacological aspect of ectopic ossification in spinal ligament tissues. Pharmacol Ther 118: 352-358, 2008.

4. Pike JW and Christakos S: Biology and mechanisms of action of the vitamin D hormone. Endocrinol Metab Clin North Am 46: 815-843, 2017.

5. Arnold A, Dennison E, Kovacs CS, Mannstadt M, Rizzoli R, Brandi ML, Clarke B and Thakker RV: Hormonal regulation of biomineralization. Nat Rev Endocrinol 17: 261-275, 2021.

6. Nakamichi Y, Udagawa N, Suda T and Takahashi N: Mechanisms involved in bone resorption regulated by vitamin D. J Steroid Biochem Mol Biol 177: 70-76, 2018.

7. Ao T, Kikuta J and Ishii M: The effects of vitamin D on immune system and inflammatory diseases. Biomolecules 11: 1624, 2021.

8. Charoenngam N: Vitamin D and rheumatic diseases: A review of clinical evidence. Int J Mol Sci 22: 10659, 2021.

9. Harrison SR, Li D, Jeffery LE, Raza K and Hewison M: Vitamin D, autoimmune disease and rheumatoid arthritis. Calcif Tissue Int 106: 58-75, 2020

10. Welldon KJ, Findlay DM, Evdokiou A, Ormsby RT and Atkins GJ: Calcium induces pro-anabolic effects on human primary osteoblasts associated with acquisition of mature osteocyte markers. Mol Cell Endocrinol 376: 85-92, 2013.

11. Drewnowski A and Shultz JM: Impact of aging on eating behaviors, food choices, nutrition, and health status. J Nutr Health Aging 5: 75-79, 2001.

12. Takahashi N, Akatsu T, Udagawa N, Sasaki T, Yamaguchi A, Moseley JM, Martin TJ and Suda T: Osteoblastic cells are involved in osteoclast formation. Endocrinology 123: 2600-2602, 1988.

13. Li J, Sarosi I, Yan XQ, Morony S, Capparelli C, Tan HL, McCabe S, Elliott R, Scully S, Van G, et al: RANK is the intrinsic hematopoietic cell surface receptor that controls osteoclastogenesis and regulation of bone mass and calcium metabolism. Proc Natl Acad Sci USA 97: 1566-1571, 2000.
14. Boyle WJ, Simonet WS and Lacey DL: Osteoclast differentiation and activation. Nature 423: 337-342, 2003.

15. Mori T, Horibe K, Koide M, Uehara S, Yamamoto Y, Kato S, Yasuda H, Takahashi N, Udagawa N and Nakamichi Y: The vitamin $D$ receptor in osteoblast-lineage cells is essential for the proresorptive activity of $1 \alpha, 25(\mathrm{OH}) 2 \mathrm{D} 3$ in vivo. Endocrinology 161: bqaa178, 2020.

16. Sun J, Sun B, Wang W, Han X, Liu H, Du J, Feng W, Liu B, Amizuka $\mathrm{N}$ and Li M: Histochemical examination of the effects of high-dose 1,25(OH)2D3 on bone remodeling in young growing rats. J Mol Histol 47: 389-399, 2016.

17. Han X, Zhu N, Wang Y and Cheng G: 1,25(OH)2D3 inhibits osteogenic differentiation through activating $\beta$-catenin signaling via downregulating bone morphogenetic protein 2. Mol Med Rep 22: 5023-5032, 2020.

18. Yamaguchi $\mathrm{M}$ and Weitzmann $\mathrm{MN}$ : High dose 1,25(OH)2D3 inhibits osteoblast mineralization in vitro. Int J Mol Med 29: 934-938, 2012.

19. Yang D, Turner AG, Wijenayaka AR, Anderson PH, Morris HA and Atkins GJ: 1,25-Dihydroxyvitamin D3 and extracellular calcium promote mineral deposition via NPP1 activity in a mature osteoblast cell line MLO-A5. Mol Cell Endocrinol 412: 140-147, 2015.

20. Misgar RA, Sahu D, Bhat MH, Wani AI and Bashir MI: Vitamin D toxicity: A prospective study from a tertiary care centre in Kashmir Valley. Indian J Endocrinol Metab 23: 363-366, 2019.

21. Abhishek A and Doherty M: Pathophysiology of articular chondrocalcinosis-role of ANKH. Nat Rev Rheumatol 7: 96-104, 2011.

22. Heneghan C and Mahtani KR: Vitamin D does not prevent fractures and falls. BMJ Evid Based Med 24: 147-148, 2019.

23. Chiodini I and Gennari L: Falls, fractures and vitamin D: A never-ending story? Nat Rev Rheumatol 15: 6-8, 2019.

24. Gallagher JC: Vitamin D and bone density, fractures, and falls: The end of the story? Lancet Diabetes Endocrinol 6: 834-835, 2018.

25. Bolland MJ and Grey A: A case study of discordant overlapping meta-analyses: Vitamin D supplements and fracture. PLoS One 9: e115934, 2014.

26. Burt LA, Billington EO, Rose MS, Kremer R, Hanley DA and Boyd SK: Adverse effects of high-dose vitamin D supplementation on volumetric bone density are greater in females than males. J Bone Miner Res 35: 2404-2414, 2020.

This work is licensed under a Creative Commons Attribution-NonCommercial-NoDerivatives 4.0 International (CC BY-NC-ND 4.0) License. 